\title{
"Alle, die mit Gruppen arbeiten, kennen den Zauber der Gruppe" - über Schreibcoaching in Gruppensettings und Teilearbeit im Einzelcoaching: Ein Praxisdialog mit Alexandra Peischer
}

\author{
Alexandra Peischer ${ }^{1} \cdot$ Melanie Fleischhacker ${ }^{2} \cdot$ Karin Wetschanow $^{2} \cdot$ Carmen Mertlitsch $^{2}$
}

Angenommen: 13. November 2020 / Online publiziert: 11. Dezember 2020

๑) Der/die Autor(en) 2020

Im Folgenden gibt das Herausgeberinnenteam des Themenschwerpunktes „Interventionen im Schreibcoaching“ (Melanie Fleischhacker, Karin Wetschanow und Carmen Mertlitsch) einer Expertin des Schreibcoachings im Einzelund Gruppensetting die Möglichkeit, von ihren Erfahrungen zu berichten. Sie wurde eingeladen in schriftlicher Form u.a. auf Fragen zum Thema ,Schreibcoaching für Gruppen“ zu antworten. Ziel dieser Darstellungsform ist es, die engen Beziehungen zwischen Wissenschaft und Praxis aufzuzeigen und den Stellenwert praktischer Expertise für eine Etablierung und theoretische Weiterentwicklung des Feldes aufzuzeigen. Mit dem Expertinneninterview wird dem praxisorientierten Ursprung der Schreibcoachingforschung Tribut gezollt und dadurch nicht nur der Wissenschaft eine Stimme gegeben.

\section{Wie definieren Sie aus Ihrer Sicht "Schreibcoaching" - auch in Abgrenzung zur Schreibberatung?}

Ich sehe Schreibcoaching als eine spezielle Form von Coaching, die auf die Begleitung von Schreibenden und deren Schreibprozessen abzielt und zwischen Fach- und Prozessberatung angesiedelt ist: Der*die Schreibcoach bringt das nötige Handwerkszeug und Fachwissen aus der Expert*innen-/Fachberatung ein, also Wissen, Methoden und Techniken aus Schreibforschung, Schreibdidaktik

\footnotetext{
Alexandra Peischer

peischer@schreibraum.com

Melanie Fleischhacker

Melanie.Fleischhacker@aau.at

schreib.raum, Claudiastraße 20, 6020 Innsbruck, Österreich

2 SchreibCenter, Universität Klagenfurt, Universitätsstraße 65-67, 9020 Klagenfurt am Wörthersee, Österreich
}

und anderen relevanten Fachbereichen und arbeitet dabei mit einer professionellen Coachinghaltung aus der (systemischen) Prozessberatung, d.h. neutrale Außensicht, Augenhöhe, Impulsgeber*in und Begleiter*in.

Schreibberatung ist in meinen Augen Fachberatung: Es wird hauptsächlich Know-how zum Schreibprozess vermittelt, Ratsuchende werden gezielt und punktuell $\mathrm{zu}$ ihren Schwierigkeiten beraten.

Schreibberatung ist auf das Produkt gerichtet, Schreibcoaching nimmt den Prozess in den Blick und damit auch das Schreiben ganz allgemein, u.U. auch einen vorhandenen Schreib-Wunsch. Schreibberatung zielt damit auf ein bestmögliches schriftliches Ergebnis, einen gelungenen Text. Schreibcoaching hingegen hat die Person des Schreibenden im Fokus. Da kann es schon auch einmal vorkommen, dass im Laufe des Coachingprozesses ein Schreibprojekt bewusst fallen gelassen wird. Schreibcoaching ist also ergebnisoffener als Schreibberatung.

In der Praxis verschwimmen diese Grenzen gelegentlich. Wesentlich beim Schreibcoaching ist für mich einerseits die Prozessbegleitung, andererseits die Anwendung klassischer Coachingtools, vor allem dann, wenn die Schwierigkeiten beim Schreiben tiefer liegen als nur im fehlenden Knowhow zum Schreiben.

Meine persönliche (zusammenfassende) Definition Schreibcoaching meint die professionelle Begleitung von Schreibenden und ihren Schreibprozessen. Schreibcoaching geht über eine reine Schreibberatung (Fachberatung, punktuelle Hilfestellung) hinaus, indem es einen Prozess initiiert und begleitet, klassische Coachingtools miteinbezieht und ganzheitlich auf den*die Coachee schaut - unter Einbeziehung des relevanten Systems, der räumlichen und sozialen Gegebenheiten, der einflussnehmenden, mitunter auch persönlichen Themen und Probleme. Schreibcoaching gibt Hilfe zur Selbsthilfe. 


\section{Welche Faktoren beeinflussen das Schreibcoaching? Worauf sollte eine Person, die Schreibcoachings abhält, vorbereitet sein?}

Coachs arbeiten immer entlang der Bedürfnisse ihrer Coachees. Die systemische Arbeitsweise berücksichtigt dabei nicht nur das Problem per se, das Symptom, sondern berücksichtigt auch Kontext, soziales Umfeld, Rahmenbedingungen und die Wechselwirkungen.

Wesentlicher Erfolgsfaktor für jeden Schreibcoachingprozess ist die wohlwollende und wertschätzende Präsenz der Coachs und eine ungetrübte Aufmerksamkeit, ja Neugier für das, was sich im Coaching bzw. bei den Coachees zeigt - ausgesprochen oder unausgesprochen. Mit einer Vielzahl von Interventionsmöglichkeiten und der nötigen Erfahrung können Coachs situationsabhängig und individuell darauf reagieren.

\section{Worin sehen Sie Chancen, aber auch Grenzen eines Gruppensettings im Schreibcoachingprozess?}

Alle, die mit Gruppen arbeiten, kennen den „Zauber der Gruppe“: Diese besondere Atmosphäre, die unterstützende Energie, die Synergien, die sich in der Gruppe entfalten. Wir kennen die positive Wirkung aus therapeutischen Kontexten genauso wie aus Selbsterfahrungs- und Lerngruppen. In meiner Praxis hat sich auch beim Schreibcoaching das Gruppensetting sehr bewährt, insbesondere im studentischen Kontext. Schon die Erfahrung, nicht alleine zu sein im Schreibprozess, motiviert: „Gemeinsam statt einsam“ geht vieles leichter, auch das Schreiben. Wenn die Teilnehmer*innen dann auch noch bemerken, dass sie nicht die Einzigen sind mit einem Schreibproblem, dass alle mit ähnlichen Schwierigkeiten umzugehen haben, steigt die $\mathrm{Zu}$ versicht, diese Hindernisse zu meistern.

Nicht geeignet ist das Gruppensetting lediglich für Personen, die sehr viel Unterstützung brauchen oder ein sehr spezielles Schreibprojekt verfolgen.

\section{Könnten Sie den Ablauf eines Schreibcoachings im Gruppensetting (mit 4-8) Personen für längere Schreibprozesse beschreiben?}

Die Gruppe trifft sich ca. 14-tägig über einen längeren Zeitraum hinweg, meist für 3 Monate. Manche Teilnehmende kommen danach für einen zweiten „Zyklus“. Innerhalb dieser Zeitspanne bleiben die Gruppen konstant, für die einzelnen Treffen empfiehlt sich eine Dauer von etwa $3 \mathrm{~h}$.
Ähnlich wie im Einzelsetting variieren auch im Gruppensetting die Inhalte, abhängig von den Wünschen und Bedürfnissen der Teilnehmenden. Der Ablauf der einzelnen Treffen orientiert sich an Modellen der Gruppensupervision (Peischer 2010; Rappe-Giesecke 2009; Schreyögg 2004).

In meiner Praxis hat sich folgende Vorgehensweise bewährt: Der erste Termin legt den Grundstein für eine vertrauensvolle und effiziente Arbeitsatmosphäre und dafür, dass alle Teilnehmenden ihren Platz in der Gruppe finden: Unterstützt durch entsprechende Methoden gebe ich ausreichend Zeit und Raum für das Kennenlernen, die Klärung von Wünschen, Erwartungen und Ängsten sowie das gemeinsame Erarbeiten von „Gruppen-Regeln“.

Die Folgetermine beginnen jeweils mit einer Anfangsrunde, in der alle zu Wort kommen, über ihre Fortschritte seit dem letzten Treffen berichten und aktuelle Fragen und Anliegen in die Gruppe bringen. Danach wird gemeinsam entschieden, wie, wie lange und in welcher Reihenfolge die Themen behandelt werden. Ein Anliegen nach dem anderen wird bearbeitet. In einer Abschlussrunde benennt schließlich jede*r Einzelne noch einmal seine*ihre Erkenntnisse und nötige nächste Schritte.

Beim letzten Termin geht es abschließend noch um die Reflexion des Gesamtprozesses und den Abschied voneinander.

\section{Was ist der Nutzen der Gruppe in einem solchen Setting? Wie lernen die Coachees voneinander?}

In der Gruppenkonstellation findet eine Bündelung von theoretischem Wissen und praktischen Erfahrungen aller Teilnehmenden statt, wodurch enormes Potenzial freigesetzt wird. Jede Person, die an der Gruppe teilnimmt, ist „Expert*in“ und bringt „Erfahrungen, Fähigkeiten, Fertigkeiten, theoretisches Wissen, Empathie ein, deren Gesamtheit in der Gruppe zu einem Zusammenwirken führt" (Petzold und Lemke 1979, S. 91). Durch die CoKreation werden hilfreiche Strategien entwickelt und Ideen vervielfacht.

Nicht zuletzt ist das Gruppensetting der ideale Ort für (Text-)Feedback. Ein angeleitetes, wertschätzend-konstruktives Feedback auf kurze Textabschnitte, Gliederungsentwürfe oder Ideen ermöglicht wahre Quantensprünge im Schreibprozess, die Teilnehmenden erwerben sowohl Schreib- als auch Feedback-Kompetenz. 


\section{Wie sehen Sie die Rolle des*r Coach im Gruppensetting im Gegensatz zum Einzelsetting? Welche Dynamiken sind dabei zu berücksichtigen?}

Die Komplexität im Gruppensetting ist naturgemäß um ein Vielfaches höher als im Einzelsetting. Der*die Coach trägt Verantwortung sowohl für die Gruppe als Ganzes als auch für jede einzelne Person, die teilnimmt, sowie für die eingebrachten Themen und deren Bearbeitung (Langmaack 2001).

Coachs achten auf eine faire und ausgeglichene Verteilung der Rede-, Beratungs- oder Feedback-Zeit pro Teilnehmer*in und auf die Beteiligung aller Gruppenmitglieder, auch wenn sie gerade mit einzelnen Teilnehmenden arbeiten. Die jeweils nicht unmittelbar Beteiligten sind aktive Zuhörer*innen oder arbeiten innerlich an einem eigenen Thema mit. So profitieren alle Teilnehmenden von allen Interventionen.

Besonders gut gelingt die Beteiligung, wenn Schreibübungen als Intervention genutzt werden. Folgendes Beispiel soll dies verdeutlichen: Ein Gruppenmitglied kämpft gerade mit der Konkretisierung der Forschungsfrage oder der Eingrenzung des Themas. Ich erkläre daraufhin den „Fokussprint“ und die „Schreibstaffel“ (Scheuermann 2012) und rege an, dass alle einen Fokussprint schreiben mit der Fragestellung: „Was finde ich spannend an meinem Thema?" (ggf. einer Frage, die noch besser zum individuellen Schreibprozess passt). Alle Teilnehmenden schreiben 5 min lang, werten das Geschriebene abschlieBend aus und formulieren einen Kernsatz. Im Anschluss liest der Falleinbringer seinen Kernsatz vor und/oder erzählt, ob bzw. was klarer geworden ist. Zum Schluss teilen auch die anderen ihre Erfahrungen und Erkenntnisse. Auf diese Weise sieht der*die Coach sehr schnell, welche Prozesse bei den Einzelnen ablaufen und an welchem Punkt des Prozesses sie jeweils stehen. Als „Hausübung“ kann mit diesem Kernsatz dann weitergeschrieben, eine sogenannte „Schreibstaffel“" absolviert werden.

\section{Welche konkreten Interventionen haben sich für Sie bisher bewährt? Welche Instrumente setzen Sie gerne ein?}

Zusätzlich zu den schreibdidaktischen und -pädagogischen Interventionen können alle klassischen Coaching- und Supervisions-Tools sowohl im Einzel- als auch im Gruppensetting verwendet werden. Für Schreibcoaching haben sich für mich systemische oder gestaltpädagogische Methoden besonders bewährt wie z. B. ressourcen- und lösungsorientierte Fragen und Techniken (Radatz 2006; Kindl-Beilfuß 2015) sowie diverse Übungen aus dem kreativen Schreiben.
Hier wird der Nutzen des Schreibens gleich doppelt erlebt: Einerseits als allgemeiner Nutzen der jeweiligen Schreibübung, andererseits erfahren Coachees das Schreiben selbst als positiv, können an Ort und Stelle mit dem*r Schreibcoach die Technik(en) reflektieren und den Einsatz für das eigene Schreibprojekt besprechen.

Besonders gern setze ich eine Übung aus der Gestaltarbeit ein, das „Treffen mit dem*r inneren Ratgeber*in“: Eine Phantasiereise führt den*die Coachee in eine selbstgewählte Landschaft, wo er*sie einen Weg entlang geht. Irgendwann begegnet ihm*r eine wohlgesonnene Person, die zu allen Anliegen befragt werden kann. Das alles wird schreibend ausgeführt und im anschließenden Gespräch nachbearbeitet. Die Übung fördert verdeckte Ressourcen und hilft bei Selbstzweifeln oder persönlichen Fragen zum Schreiben.

\section{Wie setzen Sie die tiefgehende Teilearbeit im Schreibcoaching ein? Können Sie uns dazu auch ein Fallbeispiel aus Ihrer Praxis schildern?}

Wenn ein*e Coachee starke Ambivalenzen in Bezug auf ihr*sein Schreibprojekt äußert, kann die Arbeit mit den inneren Stimmen bzw. dem ,inneren Team“, wie Schulz von Thun es nennt, hilfreich sein. Der*die Coachee soll seine*ihre inneren Teammitglieder in einem schriftlichen Dialog miteinander sprechen lassen, eine ,Teamsitzung“ einberufen bzw. mit ausgewählten Stimmen ein „Mitarbeitergespräch" führen. Falls diese Schreibaufgabe nicht im Laufe der Coachingstunde möglich ist, gebe ich sie als Hausübung mit. In der nächsten Sitzung wird dann ggf. damit weiter gearbeitet.

Ein Fallbeispiel Herbert (Name geändert), Akademiker Mitte Fünfzig, kommt ins Coaching, weil er mit seiner Abschlussarbeit für die Psychotherapieausbildung nicht weiterkommt. Genaugenommen hat er noch gar nicht angefangen. Obwohl ihm nur noch die schriftliche Arbeit für den Abschluss fehlt und er grundsätzlich keine Schwierigkeiten mit Schreiben hat, schiebt er das Anfangen Tag für Tag, Woche für Woche auf. Er versteht sich selbst nicht mehr und kommt daher ins Schreibcoaching.

Als er seine Lage schildert, höre ich viele Für- und Wider-Stimmen in ihm: „Ich möchte das endlich erledigen“, aber „eigentlich brauche ich den Abschluss ja gar nicht, ich habe die Ausbildung ja nur für mich gemacht“. „Das Thema interessiert mich sehr, da kenne ich mich auch schon gut aus“. Andererseits ,,ist der Betreuer der Arbeit so ein Theoretiker und ,I-Tüpftler", dass er sicher alles mögliche auszusetzen hat ..." So geht das weiter, bis ich ihm vorschlage, bewusst mit diesen vielen ambivalenten Stimmen 
zu arbeiten. Er stimmt zu und ich erkläre ihm kurz das Modell des „Inneren Teams“, zeichne eine große leere Silhouette aufs Flipchart, über der sein Name steht und bitte ihn, jetzt noch einmal der Reihe nach laut und verkürzt auszusprechen, welche Stimmen da in ihm sprechen. Ich zeichne bzw. schreibe mit: Jede Stimme erhält ein kleines Personen-Symbol mit einer Sprechblase, in der die wichtigste Aussage steht. Dann frage ich nach einem Namen für diese Stimme und schreibe sie unter die Figur. Wenn es ein Symbol gibt, zeichne ich das ebenfalls dazu.

Als die Stimmen nachlassen, schauen wir uns das Flipchart an: Welche Stimme ist hilfreich, welche hinderlich? Welche Stimmen verbünden sich, welche kämpfen gegeneinander? Was haben die Stimmen zueinander zu sagen?

Schnell zeigt sich, dass alle Stimmen, die ihn blockieren, in einem Eck „heimisch“ sind. Sie sind zwar nicht die lautesten, haben aber zusammen eine spürbare Macht. Gemeinsam generieren sie einen enormen Widerstand, der sich vor allem gegen die Autorität des Betreuers richtet. Das war Herbert vorher so nicht aufgefallen, es bringt ihn zum Lachen. Dass dieser Widerstand ihn dermaßen lähmt, hätte er nicht gedacht. Jetzt ist es sicht- und spürbar.

Gemeinsam überlegen wir nun, wie der Widerstand gezähmt werden könnte oder ob die Kraft des Widerstands eventuell in eine hilfreiche Kraft umgelenkt werden kann. Ihm kommt die Idee, dass diese widerständischen Stimmen sich mit seinem Selbstbewusstsein und dem Wissen um seine inhaltliche und schreiberische Kompetenz verbünden können und damit zu einer Stimme werden, die ihm den Rücken stärkt und ihm zuruft: „Jetzt erst recht! Dem zeige ich, dass ich ihm gewachsen bin.“ „Wenn er meint, er muss an meiner Arbeit noch was aussetzen, dann soll er doch. Das ist wohl auch Teil seiner Aufgabe als Betreuer.“

Herbert kann dem Betreuer jetzt zugestehen, Änderungsvorschläge zu machen. Das gibt ihm so viel Motivation und Zuversicht, dass er gestärkt nach Hause geht und mir zwei Monate später schreibt, dass er die Arbeit abgeben hat und sie (ohne Änderungswünsche!) angenommen wurde.

\section{Wenn Sie sich als Praktikerin von der Forschung etwas wünschen könnten, welche Bereiche würden Sie gerne erforscht sehen? Was wären für die Praxis sinnvolle Fragestellungen bzw. Forschungsbereiche?}

In den letzten Jahren hat sich die Forschung vermehrt mit Schreiben und Schreibprozessen, auch mit Schreibberatung und -coaching auseinandergesetzt. Das ist sehr positiv und weist in die richtige Richtung. An den Universitäten selbst könnte m.E. noch mehr geforscht werden: Welche konkreten Angebote für Schreibberatung und -coaching gibt es an den Hochschulen und wie wirken sie sich aus? Von welchen Studierenden werden die Angebote am besten angenommen bzw. am dringendsten gebraucht (Erstsemestrige, Studierende im Rahmen ihrer Abschlussarbeit, bestimmte Studienrichtungen etc.)? Gibt es Hemmschwellen zur Nutzung der Schreibberatung? Wenn ja, wie können diese überwunden werden? Was konkret wünschen sich Studierende in Bezug auf ihre Schreibentwicklung? Auch die Frage, wie Lehrende ihre Studierenden in Punkto Schreiben bestmöglich unterstützen können und was sie dazu brauchen (an Know-how, Rahmenbedingungen etc.), wäre ein interessanter Forschungsbereich.

Open Access Dieser Artikel wird unter der Creative Commons Namensnennung 4.0 International Lizenz veröffentlicht, welche die Nutzung, Vervielfältigung, Bearbeitung, Verbreitung und Wiedergabe in jeglichem Medium und Format erlaubt, sofern Sie den/die ursprünglichen Autor(en) und die Quelle ordnungsgemäß nennen, einen Link zur Creative Commons Lizenz beifügen und angeben, ob Änderungen vorgenommen wurden.

Die in diesem Artikel enthaltenen Bilder und sonstiges Drittmaterial unterliegen ebenfalls der genannten Creative Commons Lizenz, sofern sich aus der Abbildungslegende nichts anderes ergibt. Sofern das betreffende Material nicht unter der genannten Creative Commons Lizenz steht und die betreffende Handlung nicht nach gesetzlichen Vorschriften erlaubt ist, ist für die oben aufgeführten Weiterverwendungen des Materials die Einwilligung des jeweiligen Rechteinhabers einzuholen.

Weitere Details zur Lizenz entnehmen Sie bitte der Lizenzinformation auf http://creativecommons.org/licenses/by/4.0/deed.de.

\section{Literatur}

Kindl-Beilfuß, C. (2015). Fragen können wie Küsse schmecken. Systemische Fragetechniken für Anfänger und Fortgeschrittene, 6. Aufl. Heidelberg: Carl Auer.

Langmaack, B. (2001). Einführung in die Themenzentrierte Interaktion TZI. Leben rund ums Dreieck. Weinheim/Basel: Beltz.

Peischer, A. (2010). Supervision für Studierende. Einsatzmöglichkeiten und Chancen eines bewährten Beratungsformates in einem neuen Kontext. Innsbruck: Studienverlag.

Petzold, H., \& Lemke, J. (1979). Gestaltsupervision als Kompetenzgruppe. Gestalt-Bulletin, 3(1), 88-94.

Radatz, S. (2006). Einführung in das systemische Coaching. Heidelberg: Carl Auer.

Rappe-Giesecke, K. (2009). Supervision für Gruppen und Teams, 4. Aufl. Berlin/Heidelberg: Springer.

Scheuermann, U. (2012). Schreibdenken. Schreiben als Denk- und Lernwerkzeug nutzen und vermitteln. Opladen/Toronto: Budrich/UTB.

Schreyögg, A. (2004). Supervision. Ein integratives Modell. Lehrbuch zu Theorie und Praxis, 4. Aufl. Wiesbaden: Verlag für Sozialwissenschaften. 


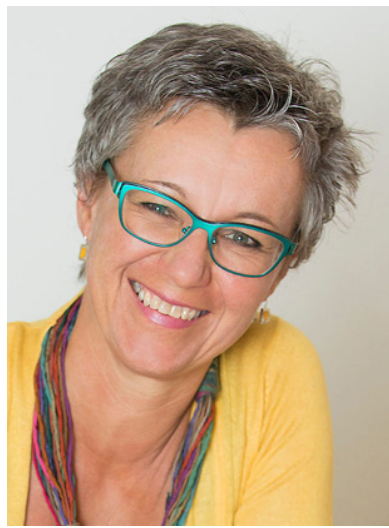

Alexandra Peischer ist gelernte Buchhändlerin und Bildungswissenschaftlerin und arbeitet seit 2008 als selbständige Supervisorin und Coach, Gestaltpädagogin, Lebensund Sozialberaterin sowie (Schreib)trainerin, Schreib(päd)agogin und Yogalehrerin. In ihrer Arbeit verbindet sie diese verschiedenen Ausbildungen miteinander, insbesondere das systemische Coaching, die gestaltpädagogische Grundhaltung und das kreative Schreiben. In dem 2012 von ihr gegründeten „schreib.raum für studium, beruf und freizeit" in Innsbruck bietet sie Schreibcoaching und eine bunte Palette an Schreibseminaren an. Seit 2017 bildet sie selbst Schreibagog*innen und Schreibcoachs aus: "Schreibagogik ${ }^{\circledR}$ I - Schreiben(d) begleiten“ vermittelt Basiskenntnisse für alle, die mit Schreiben arbeiten wollen, der Aufbaulehrgang „Schreibagogik ${ }^{\circledR}$ II - Schreibcoaching“" vertieft die Kompetenz zur*m Schreibcoach. 